\title{
Dynamical Evolution, Hadronization and Angular De-correlation of Heavy Flavor in a Hot and Dense QCD Medium
}

\author{
Shanshan $\mathrm{CaO}^{\mathrm{a}}$, Guang-You Qin ${ }^{\mathrm{b}}$, Steffen A Bass ${ }^{\mathrm{a}}$ \\ ${ }^{a}$ Department of Physics, Duke University, Durham, NC 27708, USA \\ ${ }^{b}$ Institute of Particle Physics and Key Laboratory of Quark and Lepton Physics (MOE), Central China Normal University, Wuhan, 430079, China
}

\begin{abstract}
We study heavy flavor evolution and hadronization in relativistic heavy-ion collisions. The in-medium evolution of heavy quarks is described using our modified Langevin framework that incorporates both collisional and radiative energy loss mechanisms. The subsequent hadronization process for heavy quarks is calculated with a fragmentation plus recombination model. We find significant contribution from gluon radiation to heavy quark energy loss at high $p_{\mathrm{T}}$; the recombination mechanism can greatly enhance the $D$ meson production at medium $p_{\mathrm{T}}$. Our calculation provides a good description of the $D$ meson nuclear modification at the LHC. In addition, we explore the angular correlation functions of heavy flavor pairs which may provide us a potential candidate for distinguishing different energy loss mechanisms of heavy quarks inside the QGP.
\end{abstract}

Keywords: heavy flavor, energy loss, hadronization, correlation function

\section{Introduction}

Heavy quarks serve as excellent probes of the highly excited and color deconfined QCD matter - known as the Quark-Gluon Plasma (QGP) - created in ultrarelativistic heavy-ion collisions. They are mainly produced at the primordial stage of the collisions and then propagate through and probe the whole evolution history of the QGP fireballs. Because of their large masses, heavy quarks were usually expected to interact more weakly with the medium than light partons. However, experimental data from at both RHIC and the LHC have revealed surprisingly small values of $R_{\mathrm{AA}}$ and large values of $v_{2}$ for heavy flavor mesons and their decay electrons [1-4]. Various theoretical frameworks have been constructed to explore this "heavy flavor puzzle", such as the Boltzmann-based parton cascade model [5], the linearized Boltzmann transport of heavy quarks inside a hydrodynamic medium [6], and the Langevin evolution of heavy quarks inside QGP [7].

We follow our previous work [7-10] and study the dynamical evolution and hadronization of heavy quark in heavyion collisions. The energy loss of heavy quarks in QGP medium is described using a modified Langevin equation that simultaneously incorporates mechanisms of quasi-elastic scattering and medium-induced gluon radiation. The subsequent hadronization process is calculated with a hybrid fragmentation plus recombination model. Within this new framework, we demonstrate that while collisional energy loss dominates the heavy flavor nuclear modification in the low $p_{\mathrm{T}}$ region, gluon radiation dominates at high $p_{\mathrm{T}}$. The recombination mechanism may significantly enhance the production of heavy mesons at medium $p_{\mathrm{T}}$. Our calculation provides a good description of $D$ meson $R_{\mathrm{AA}}$ as measured by the ALICE collaboration. We also study the angular correlation functions of heavy flavor pairs and find them sensitive to different energy loss mechanisms. 


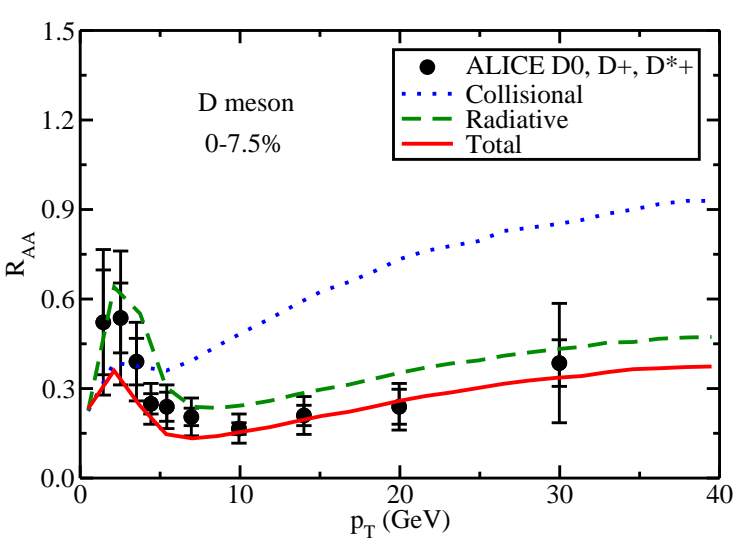

Figure 1. (Color online) Comparison of $D$ meson $R_{\mathrm{AA}}$ between different energy loss mechanisms.

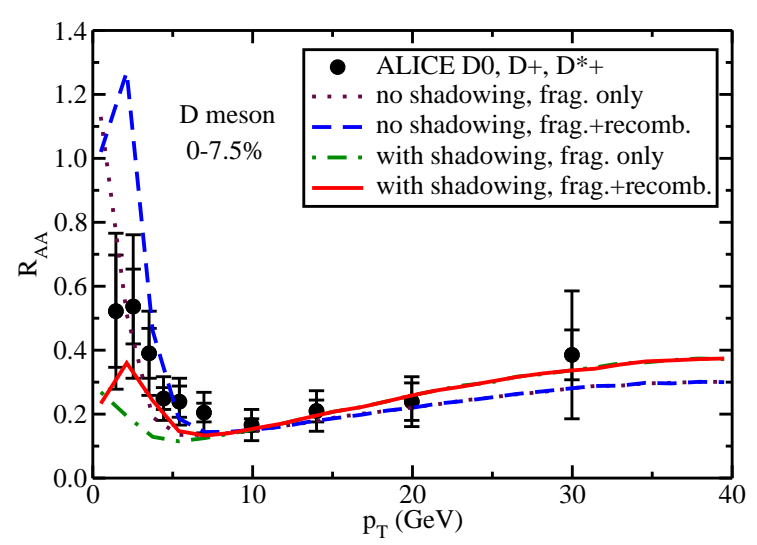

Figure 2. (Color online) Comparison between different initialization and hadronization methods. This figure is taken from Ref. [7]

\section{In-medium Evolution and Hadronization of Heavy Quarks}

In the limit of multiple scatterings, the dynamical evolution of heavy quarks inside a QGP can be treated as the Brownian motion that is typically described by the Langevin equation. To incorporate the effects from both quasielastic scatterings and medium-induced gluon radiation, we modify the classical Langevin equation as follows:

$$
\frac{d \vec{p}}{d t}=-\eta_{D}(p) \vec{p}+\vec{\xi}+\vec{f}_{g}
$$

Apart from the first two terms on the right hand side that represent the drag and the thermal random forces, a recoil force term $\vec{f}_{g}=-d \overrightarrow{p_{g}} / d t$ is introduced to describe the effect of gluon radiation on heavy quark motion. The probability of gluon radiation during each time interval $\Delta t$ and the momentum of radiated gluon $\left(\Delta \overrightarrow{p_{g}}\right)$ are simulated using the Monte-Carlo method according to the following equation, taken from the Higher-Twist energy loss formalism [11]:

$$
\frac{d N_{g}}{d x d k_{\perp}^{2} d t}=\frac{2 \alpha_{s}\left(k_{\perp}\right)}{\pi} P(x) \frac{\hat{q}}{k_{\perp}^{4}} \sin ^{2}\left(\frac{t-t_{i}}{2 \tau_{f}}\right)\left(\frac{k_{\perp}^{2}}{k_{\perp}^{2}+x^{2} M^{2}}\right)^{4},
$$

where $\hat{q}$ is the gluon transport coefficient, $k_{\perp}$ is the gluon transverse momentum, $x$ is the fractional energy carried by radiated gluon, $\tau_{f}$ is the gluon formation time, and $P(x)$ is the splitting function. $t_{i}$ represents the initial time that is reset to be the gluon emission time every time a gluon is produced so that the probability for gluon radiation starts to accumulate again. The fluctuation-dissipation relation between the drag and the thermal force $-\eta_{D}(p)=\kappa /(2 T E)$ still holds, where $\kappa$ is the momentum space diffusion coefficient defined in $\left\langle\xi^{i}(t) \xi^{j}\left(t^{\prime}\right)\right\rangle=\kappa \delta^{i j} \delta\left(t-t^{\prime}\right)$. In principle, $\kappa$ may be different in transverse and longitudinal directions, but here we assume it to be isotropic for a minimal model. For radiative process, we set a lower cutoff $\omega_{0}=\pi T$ for gluon energy to incorporate the balance between emission and absorption. Different transport coefficients are related via $D=2 T^{2} / \kappa$ and $\hat{q}=2 \kappa C_{A} / C_{F}$, where $D$ is the spatial diffusion coefficient of the heavy quark in the QGP. For the results presented in this section, the diffusion coefficient is set as $D=6 /(2 \pi T)$.

We use our modified Langevin framework to simulate the heavy quark evolution inside the QGP. The initial momentum distributions of heavy quarks are calculated using the leading-order pQCD approach unless otherwise specified. To calculate the partonic cross sections, we adopt CTEQ parton distribution functions [12] and include the nuclear shadowing effect using the EPS08 parametrization [13]. After heavy quarks traverse the medium, their hadronization is simulated according to a hybrid fragmentation plus coalescence model developed in Ref. [7]. In our work, the QGP is simulated with a (2+1)-dimensional viscous hydrodynamic model (VISH2+1) [14-16].

In Fig.1, we compare $D$ meson $R_{\mathrm{AA}}$ between different energy loss mechanisms. One observes that collisional energy loss dominates the low $p_{\mathrm{T}}$ regime while gluon radiation dominates high $p_{\mathrm{T}}$. Our combination of the two mechanisms provides a good description of the experimental data from the ALICE collaboration. In Fig.2, we investigate 


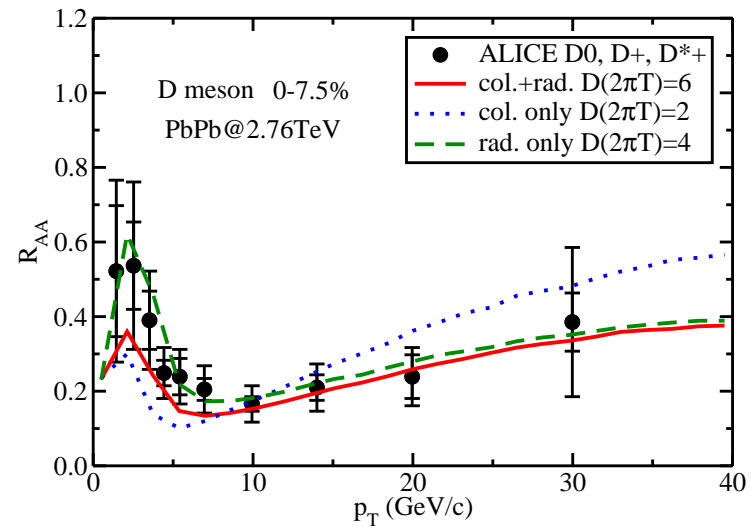

Figure 3. (Color online) Fitting $D$ meson $R_{\mathrm{AA}}$ with different energy loss mechanisms by tuning the diffusion coefficient.

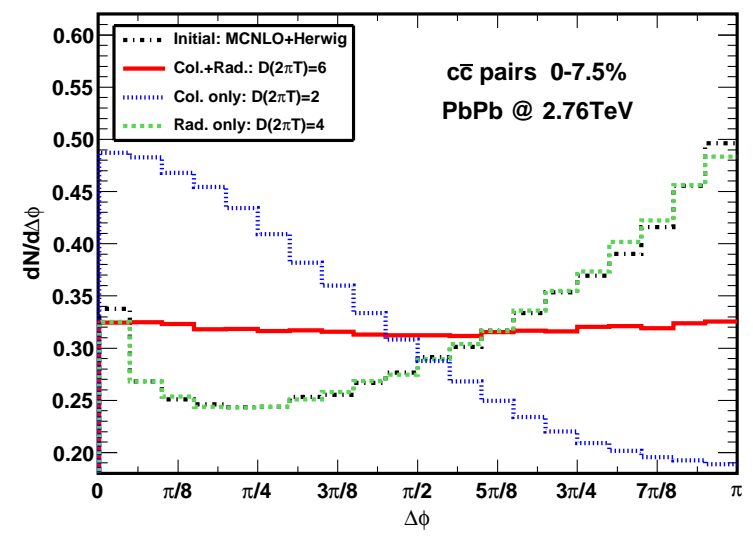

Figure 5. (Color online) Angular correlation functions of $c \bar{c}$ pairs with MCNLO+Herwig initial conditions.

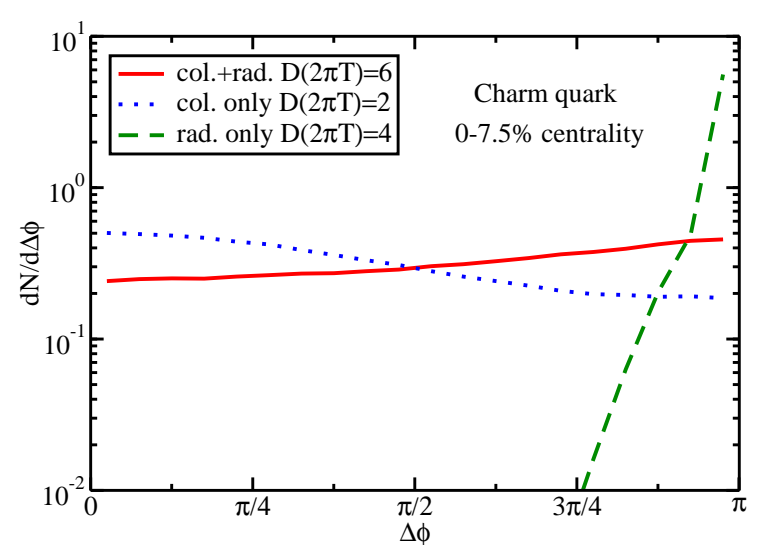

Figure 4. (Color online) Comparison of the angular correlation functions of $c \bar{c}$ pairs between different energy loss mechanisms.

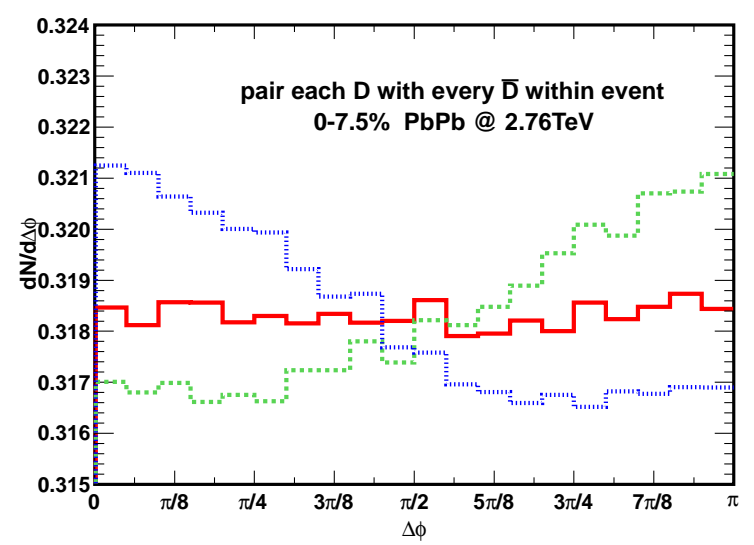

Figure 6. (Color online) Angular correlation functions of $D-\bar{D}$ with MCNLO+Herwig initial conditions.

the effects of the nuclear shadowing effect in the initial production and the recombination mechanism in the hadronization process on the heavy flavor quenching and find that the shadowing effect significantly suppress the $D$ meson $R_{\mathrm{AA}}$ at low $p_{\mathrm{T}}$ while the heavy-light quark recombination enhances the production rate of $D$ meson at medium $p_{\mathrm{T}}$. More numerical results, such as the heavy meson $v_{2}$ and comparisons with the RHIC data can be found in Ref. [7].

\section{Angular Correlation Functions of Heavy Flavor Pairs}

In the previous section, we have shown a good description of $D$ meson $R_{\mathrm{AA}}$ when incorporating both collisional and radiative energy losses for heavy quarks. One may wonder how each energy loss mechanisms alone can describe the data; this is shown in Fig.3. We can see that by tuning the transport coefficient, collisional or radiative energy loss alone also provide reasonable description of the experimental data, though not as good as the case with both energy loss mechanisms included. To distinguish between different energy loss mechanisms, one may need explore more observables for heavy flavors.

In Fig.4, we investigate the angular correlation functions of $c \bar{c}$ pairs after they propagate through a QGP medium. We start with the back-to-back production of initial $c$ and $\bar{c}$ pairs and find that after they traverse the medium, the correlation function still peaks around $\pi$ if one only considers gluon radiation. On the contrary, a peak near 0 is 
observed if one only includes collisional energy loss. This indicates that unlike the back-to-back initial state, low energy $c \bar{c}$ pairs tend to move collinearly in the end because of the boost by the radial flow of the medium.

In Fig.5, we use an improved initialization for $c \bar{c}$ production - the Monte-Carto next-to-leading-order (MCNLO) production plus Herwig vacuum radiation [6]. Similar to Fig.4, while pure gluon radiation does not change the angular correlation function compared to the initial one, pure collisional energy loss leads to a peak around 0 . A more realistic analysis is implemented in Fig.6 where we loop each $D$ meson over all $\bar{D}$ within a collision event and analyze all possible pairs. We observe that the shapes of the $D-\bar{D}$ correlation functions resemble those of $c \bar{c}$ pair in Fig.5 except for a large background contributed from uncorrelated $D$ and $\bar{D}$. These correlation functions may provide a deeper insight into heavy quark energy loss mechanisms if a comparison can be made between theory and experiment. We note that the angular correlation functions depend on details in the model calculations, such as the relation between longitudinal and transverse momentum diffusion coefficients. They also depend on experimental setups, such as selections of centrality and momentum cutoff which would be investigated in more detail in an upcoming study.

\section{Summary}

We have studied the heavy flavor evolution and hadronization in heavy-ion collisions. The quasi-elastic scatterings of heavy quarks off light partons and the medium-induced gluon radiation are simultaneously included in our modified Langevin framework. The subsequent hadronization process is calculated with a fragmentation plus recombination model. Our calculations provide a good description of the $D$ meson $R_{\mathrm{AA}}$ at the LHC. In addition, we explore the angular correlation functions of heavy flavor pairs and find them a potential candidate to distinguish different energy loss mechanisms of heavy quarks inside the QGP.

We are grateful to Prof. Berndt Müller and Dr. Marlene Nahrgang for helpful discussions, and thank the cooperations within the JET Collaboration. This work was supported by the U.S. Department of Energy Grant No. DE-FG02-05ER41367 and Natural Science Foundation of China (NSFC) under grant No. 11375072.

\section{References}

[1] A. Adare, et al., Heavy Quark Production in $p+p$ and Energy Loss and Flow of Heavy Quarks in $\mathrm{Au}+\mathrm{Au} \mathrm{Collisions}$ at $\sqrt{s_{N} N}=200 \mathrm{GeV}$, Phys. Rev. C84 (2011) 044905. arXiv:1005.1627, doi:10.1103/PhysRevC.84.044905.

[2] D. Tlusty, Open charm hadron production via hadronic decays at STAR, Nucl. Phys. A904-905 (2013) 639c-642c. arXiv:1211.5995, doi:10.1016/j.nuclphysa.2013.02.097.

[3] A. Grelli, $D$ meson nuclear modification factors in Pb-Pb collisions at $\sqrt{s_{N N}}=2.76 \mathrm{TeV}$ with the ALICE detector, Nucl. Phys. A904-905 (2013) 635c-638c. arXiv:1210.7332, doi:10.1016/j.nuclphysa.2013.02.096.

[4] D. Caffarri, Measurement of the $D$ meson elliptic flow in Pb-Pb collisions at $\sqrt{s_{N N}}=2.76$ TeV with ALICE, Nucl. Phys. A904-905 (2013) 643c-646c. arXiv:1212.0786, doi:10.1016/j.nuclphysa.2013.02.098.

[5] J. Uphoff, O. Fochler, Z. Xu, C. Greiner, Open Heavy Flavor in Pb+Pb Collisions at $\sqrt{s}=2.76$ TeV within a Transport Model, Phys. Lett. B717 (2012) 430-435. arXiv:1205.4945, doi:10.1016/j.physletb.2012.09.069.

[6] M. Nahrgang, J. Aichelin, P. B. Gossiaux, K. Werner, Azimuthal correlations of heavy quarks in $\mathrm{Pb}+\mathrm{Pb}$ collisions at $\mathrm{LHC}(\sqrt{s}=2.76$ TeV)arXiv: 1305.3823 .

[7] S. Cao, G.-Y. Qin, S. A. Bass, Heavy quark dynamics and hadronization in ultra-relativistic heavy-ion collisions: collisional versus radiative energy loss, Phys.Rev. C88 (2013) 044907. arXiv:1308.0617, doi:10.1103/PhysRevC.88.044907.

[8] S. Cao, S. A. Bass, Thermalization of charm quarks in infinite and finite QGP matter, Phys. Rev. C84 (2011) 064902. arXiv:1108.5101, doi:10.1103/PhysRevC.84.064902.

[9] S. Cao, G.-Y. Qin, S. A. Bass, Model and parameter dependence of heavy quark energy loss in a hot and dense medium, J. Phys. G40 (2013) 085103. arXiv:1205.2396, doi:10.1088/0954-3899/40/8/085103.

[10] S. Cao, G.-Y. Qin, S. A. Bass, B. Muller, Heavy quark energy loss and angular de-correlation in a quark-gluon plasma matter, J.Phys.Conf.Ser. 446 (2013) 012035. doi:10.1088/1742-6596/446/1/012035.

[11] B.-W. Zhang, E. Wang, X.-N. Wang, Heavy quark energy loss in nuclear medium, Phys. Rev. Lett. 93 (2004) 072301. arXiv:nucl-th/0309040, doi:10.1103/PhysRevLett.93.072301.

[12] H. L. Lai, et al., Global QCD analysis of parton structure of the nucleon: CTEQ5 parton distributions, Eur. Phys. J. C12 (2000) 375-392. arXiv:hep-ph/9903282, doi:10.1007/s100529900196.

[13] K. J. Eskola, H. Paukkunen, C. A. Salgado, An Improved global analysis of nuclear parton distribution functions including RHIC data, JHEP 0807 (2008) 102. arXiv:0802.0139, doi:10.1088/1126-6708/2008/07/102.

[14] H. Song, U. W. Heinz, Suppression of elliptic flow in a minimally viscous quark- gluon plasma, Phys. Lett. B658 (2008) $279-283$. arXiv:0709.0742, doi:10.1016/j.physletb.2007.11.019.

[15] H. Song, U. W. Heinz, Causal viscous hydrodynamics in 2+1 dimensions for relativistic heavy-ion collisions, Phys. Rev. C77 (2008) 064901. arXiv:0712.3715, doi:10.1103/PhysRevC.77.064901.

[16] Z. Qiu, C. Shen, U. Heinz, Hydrodynamic elliptic and triangular flow in Pb-Pb collisions at sqrt(s)=2.76ATeV, Phys. Lett. B707 (2012) 151-155. arXiv:1110.3033, doi:10.1016/j.physletb.2011.12.041. 